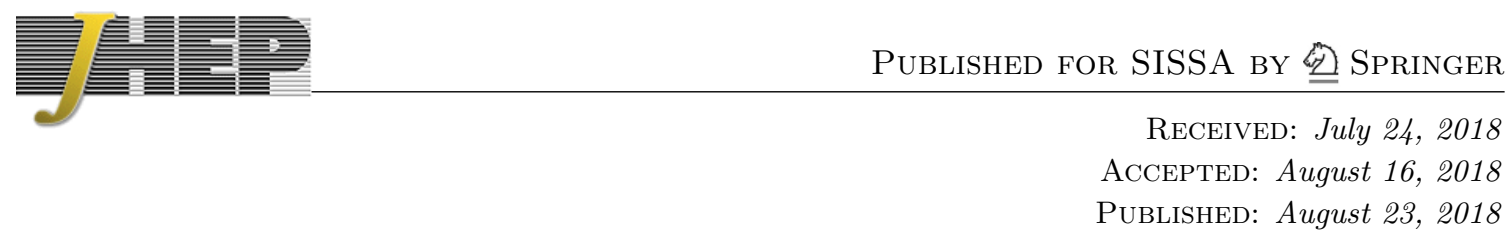

\title{
Displaced vertices from hidden glue
}

\section{Gustavo Burdman and Gabriela Lichtenstein}

Department of Mathematical Physics, Institute of Physics, University of Sao Paulo, R. do Matao 1371, Sao Paulo, SP 05508-090, Brazil

E-mail: burdman@fma.if.usp.br, gabriela.lichtenstein@usp.br

ABSTRACT: We consider the phenomenology of glueball production and decay in theories with hidden glue. We focus on the case of folded supersymmetry (FSUSY), where there is an unbroken $\mathrm{SU}(3)$ gauge theory in the absence of light matter, leading to the formation of glueballs. We study their production through the annihilation of folded squarks into hidden gluons at the LHC, and model their fragmentation into glueballs. We obtain the distribution of displaced vertices, which is directly determined by the folded squark mass scale. Although here we specifically apply it to FSUSY, the procedure to model the hidden glue fragmentation into glueballs can be generalized to other similar theories.

Keywords: Beyond Standard Model, Higgs Physics

ArXiv ePrint: 1807.03801 


\section{Contents}

1 Introduction 1

2 Hidden glue in natural extensions of the standard model 3

$\begin{array}{llr}3 & \text { Glueball fragmentation } & 6\end{array}$

4 Displaced vertices from glueballs $\quad 10$

5 Conclusions 14

\section{Introduction}

The discovery of the Higgs boson [1, 2] completes the spectrum of the standard model (SM). It also confirms its validity at the weak scale, where the gauge interactions have been tested with great precision, and now the Higgs sector responsible for electroweak symmetry breaking appears to be just as in the SM. It is clear that there are still many questions left to answer. Among them, the origin of dark matter, the large hierarchy of Yukawa couplings, the origin of neutrino masses and of the matter-anti-matter asymmetry. However, the question that potentially limits the validity of the SM the most is that of the stability of the weak scale as defined by the Higgs sector. Since in the SM the Higgs potential is not protected by any symmetry, it is highly sensitive to ultraviolet (UV) scales. This can be seen by considering the loop contributions of various SM states to $m_{h}^{2}$, resulting in quadratic sensitivity to the UV. In particular, since the top quark is the state that couples the most to the Higgs boson its contribution to $m_{h}^{2}$ determines the tuning of the Higgs mass counterterm for a given value of the UV cutoff. In order to avoid large tunings the SM cutoff should not be too far above the TeV scale. Thus, the absence of signals for physics beyond the SM constitutes a problem: the SM must be highly tuned.

Extensions of the SM that address the Higgs sector UV sensitivity do so by introducing a symmetry that protects the Higgs mass. For instance, in supersymmetric extensions [3, 4], super-partners of the SM states - particularly of the top quark - are responsible to cancel the quadratic UV sensitivity of $m_{h}^{2}$. These are the (left and right-handed) scalar partners of the top quark, the stops. On the other hand, in theories where the Higgs is a composite state its mass is typically protected by a global symmetry, which is spontaneously broken and has the Higgs as one of its pseudo-Nambu-Goldstone bosons (pNGBs) [5, 6]. In these theories, the symmetry partners of the top quark are fermions that together with the top form representations of the global symmetry group. The fact that none of these new states have been seen, either directly or through their indirect effects, puts stringent bounds on their masses, pushing the SM cutoff to higher scales. Given these bounds, these extensions of the SM are beginning to suffer their own fine-tuning problem. In particular, the bounds 
on the masses of the top quark partner make their crucial cancellation of UV sensitivity less efficient.

In most extensions of the SM mentioned above, the top partners carry SM SU(3) color. However, it is possible to significantly alleviate the tuning of extensions of the SM in models where the top partners are not charged under the SM color gauge group. These theories, grouped under the name of neutral natural models, mimic the SM extensions described above. For the case when the Higgs is a pNGB, colorless top partners are present in the Twin Higgs [7] and Quirky Little Higgs [8] models. When the symmetry protecting the Higgs mass is supersymmetry, this can be realized in Folded Supersymmetry [9]. Many more recent realizations of these ideas exist in the literature [10-19]

In Folded Supersymmetry (FSUSY), just as in the other neutral natural models, there is an unbroken $\mathrm{SU}(3)$ gauge group surviving at low energies, folded color. The folded superpartners of the SM quarks, f-squarks, are charged under this symmetry, not the SM color group. The minimal low energy theory contains the SM and the f-squarks including the f-stop responsible for stabilizing the weak scale. In addition, since the lightest matter states charged under f-color are very massive (with f-quarks in the hundreds of $\mathrm{GeV}$ ), the only light hadrons of the unbroken f-color group are f-glueballs. These states, once produced cannot be detected, with the notable exception of states that mix with the Higgs through a top partner loop. Then once produced, the lowest lying $0^{++} \mathrm{f}$-glueballs will decay back to SM states with large lifetimes, potentially resulting in highly displaced vertices. The production of f-glueballs in rare decays of the Higgs have been studied in ref. [20].

Here, we will be interested in the f-glueballs coming from the annihilation of electroweakly produced f-squark pairs. As shown in ref. [21], when f-squarks are produced they cannot hadronize and must de-excite by shedding soft radiation until they annihilate, mostly into f-gluon pairs. In turn, these will hadronize into f-glueballs [22]. Assuming that a significant fraction of these f-glueballs will be in the $0^{++}$ground state, they can decay back to the SM via higher dimensional operators. Here we are interested in calculating the distribution of displaced vertices that results from this process. For this purpose, we will need to model the fragmentation of gluons into glueballs in the hidden sector. Since the f-squark masses are much larger that the scale where the f-color interaction becomes strong, the f-glueball mass $M_{\tilde{G}}$ is expected to be considerably smaller that the f-gluon energy. Then, the fragmentation process is important in determining the pattern of displaced vertices generated by each hard f-gluon.

Although here applied to the specific case of FSUSY, the modeling of the fragmentation function we perform generally applies to theories with hidden glue where matter is much heavier than the hadronization scale. Thus, we expect that with only minor changes, the fragmentation functions found here can be used to study the displaced vertex signals of other hidden glue scenarios with glueballs such as the fraternal twin Higgs [12], the quirky little Higgs [8], the vector-like Twin Higgs [16], the hyperbolic Higgs [18], a singletscalar top partner [19]; as well as any realization of hidden valley models [23-25] where glueballs are the lightest hadron in the spectrum [26, 27]. A better understanding of the fragmentation process into glueballs in these well-motivated scenarios will allow us to test them at current experiments and even in dedicated new proposed ones [28]. 
The rest of the paper is organized as follows: in the next section we review general aspects of FSUSY and define the parameters of the theory. In section 3 we set up the phenomenology of f-squark production and its posterior annihilation into f-gluon pairs, as well as the general aspects of the glueball lifetime. In section 4 we model the fragmentation function of f-gluons into f-glueballs and obtain the distribution of displaced vertices in a variety of situations. Finally, we conclude in section 5 .

\section{Hidden glue in natural extensions of the standard model}

In this section we will focus on FSUSY in order to provide a complete example of the glueball parameters necessary to study the phenomenology of the associated hidden glue. In particular, we need to understand the relationship between the glueball mass and the matter spectrum of the theory. In FSUSY, this is given by the f-squarks.

The aim of FSUSY is to solve the little hierarchy problem, i.e. the already large gap between the weak scale and the scale of new physics that is implied by the current bounds on new particles from the LHC as well as indirect observables. In particular, since the top quark contribution to $m_{h}^{2}$ is the largest, it determines the tuning of the SM extension. In the typical weak scale supersymmetric models, this means that the stop mass cannot be too large. The tension arises from the direct bounds on squark and gluinos from the LHC, which are typically already above a TeV $[29,30]$. The way FSUSY addresses this is by having a low energy spectrum where there are squarks that are not charged under QCD, and not having gluinos. The relevant Yukawa terms are

$$
\mathcal{L} \supset\left(Y_{t} h_{u} q_{A} t_{A}+\text { h.c. }\right)+Y_{y}^{2}\left|\tilde{q}_{B} h_{u}\right|^{2}+Y_{t}^{2}\left|\tilde{t}_{B}\right|^{2}\left|h_{u}\right|^{2},
$$

where the indices $A$ and $B$ refer to the SM QCD gauge group $\mathrm{SU}(3)_{A}$ and another $\mathrm{SU}(3)_{B}$ gauge group under which the scalars are charged. In (2.1) $q_{A}$ is the third generation quark $\mathrm{SU}(2)_{L}$ doublet and $h_{U}$ is the up-type Higgs doublet. It is clear that this form of the Yukawa sector does not contribute quadratic UV sensitivity to $m_{h}^{2}$, just as is the case of typical weak scale SUSY models such as the minimal supersymmetric SM (MSSM). The crucial difference is that the scalar top partners here do not carry QCD charges and therefore can only be electroweakly produced at colliders. It is possible to obtain (2.1) in UV completions in five-dimensional (5D) theories where SUSY is broken by Scherk-Schwarz boundary conditions [9]. The gauge symmetry is $\mathrm{SU}(3)_{A} \times \mathrm{SU}(3)_{B} \times Z_{A B}$, where $Z_{A B}$ is a discrete symmetry that exchanges the $A$ and $B$ indexes. In the $5 \mathrm{D}$ construction the theory is supersymmetric in the bulk. The Scherk-Schwarz SUSY breaking implements the orbifold projection necessary to obtain the desired low energy spectrum: quarks charged under $\mathrm{SU}(2)_{A}$ and squarks charged under $\mathrm{SU}(3)_{B}$, the f-squarks. Besides the QCD-charged squarks, the orbifold procedure gets rid also of gauginos, including gluinos. Then, as advertised, we have the spectrum necessary to stabilize the little hierarchy without introducing new states charged under the SM color.

In addition to f-squarks, the minimal necessary FSUSY low energy spectrum includes an additional unbroken $\mathrm{SU}(3)_{B}$ gauge interaction. Since the lightest matter fields charged 


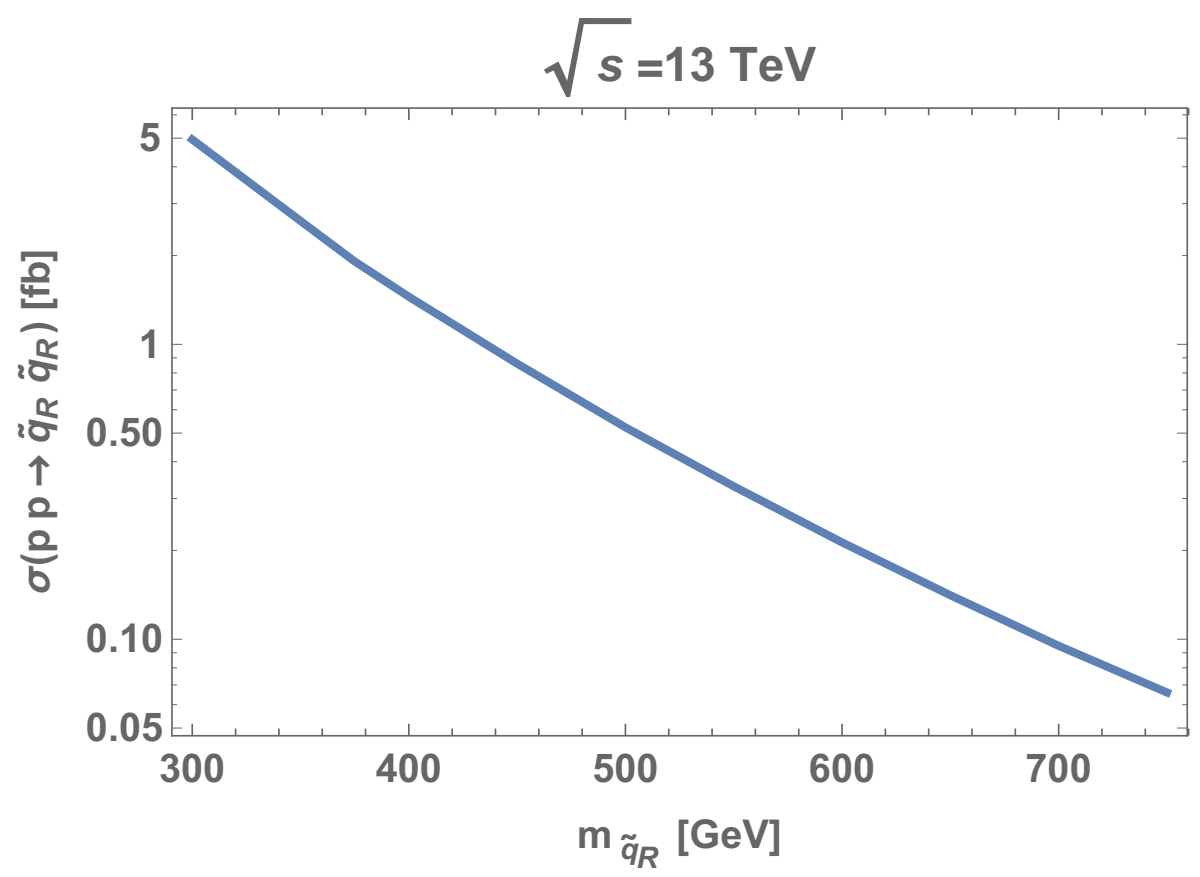

Figure 1. Cross section for the production of right-handed f-squarks vs. their mass. We assume $m_{\tilde{u}_{R}}=m_{\tilde{d}_{R}}=m_{\tilde{c}_{R}}=m_{\tilde{s}_{R}}=m_{\tilde{b}_{R}}$. See text for explanation.

under $\mathrm{SU}(3)_{B}$ are the f-squarks, which are hundreds of $\mathrm{GeV}$ (see below), the low-lying hadron spectrum of this new strong interaction is populated by glueballs.

The heavy matter charged under a new unbroken non-abelian gauge interaction, in this case $\mathrm{SU}(3)$, are generically called quirks if fermionic or squirks if scalar. Their phenomenology was studied in [31] and further in [32, 33]. The case of f-squarks was first studied in ref. [21], where it is shown that after being produced through electroweak interactions they will form a flux tube that emits soft radiation, and eventually will come back to annihilate. All of this process is prompt and results mostly into a pair of hard f-gluons, which will then hadronize into glueballs. This will be the process of interest for us in the rest of the paper. However, if we consider the production through the charged current then the preferred annihilation channel is into $W^{ \pm} \gamma$. This leads to bounds on the left-handed f-squark masses [34] which for the LHC Run I were just below $500 \mathrm{GeV}$. The right-handed f-quarks are somewhat lighter since soft masses are generated by finite loop corrections dominated by the gauge interactions [35]. We will then focus on the glueballs generated by the annihilation of these f-quarks, although much of what will do can also applied to the left-handed squarks.

The cross section for pair production of right-handed f-squarks at the LHC with $\sqrt{s}=$ $13 \mathrm{TeV}$ is shown in figure 1 as a function of the f-squark mass. In the minimal F-SUSY realization the f-squark soft masses are determined by finite loop effects [35], mainly from gauge interactions. Only the third generation doublet and the right-handed top f-squarks are significantly affected by Higgs loops. Assuming this holds in more general scenarios we take

$$
m_{\tilde{u}_{R}}=m_{\tilde{d}_{R}}=m_{\tilde{c}_{R}}=m_{\tilde{s}_{R}}=m_{\tilde{b}_{R}}
$$


only excluding the right-handed f-stop. Furthermore, the left-handed f-squarks are heavier due to the additional contributions coming form the $\mathrm{SU}(2)_{L}$ sector [9]. So here we consider the production of the lighter degenerate right-handed f-squark sector excluding the f-stop. This will give us a baseline to obtain estimates of glueball production.

Once pair-produced, the f-squarks fly apart but they cannot hadronize due to the absence of light f-colored matter. The ensuing process of de-excitation has been studied in detail in ref. [21]. After shedding some soft electromagnetic and f-glueball radiation, the pair forms a s-wave f-squarkonium, which then decays either to f-gluons or to a lesser extent back to SM fermions or gauge bosons. The charged pair production through an s-channel $W^{ \pm}$was studied in [34]. In this case the left-handed f-quarks decay back preferentially to $W^{ \pm} \gamma$, resulting in bounds on $m_{\tilde{q}_{L}}$. The current bounds vary from $320 \mathrm{GeV}$ to $465 \mathrm{GeV}$ extracted from the $8 \mathrm{TeV}$ ATLAS data [36], where the range corresponds to considering one to three generations of left-handed f-squarks.

Right-handed f-squarks are expected to be lighter. However, since there main decay mode is into glueballs, at the moment we lack any bounds on their masses. In these cases the annihilation of f-squarks after they are pair-produced and they come back together, is expected to overwhelmingly go into a pair of f-gluons, with each of them hadronizing to a number of glueballs. Below we will model this hadronization process. Once produced, folded glueballs would escape detection unless they decay back to SM states. This has been shown to happen through Higher dimensional operators induced by the Higgs first in ref. [12] in the context of the Fraternal Twin Higgs (FTH) model, and later applied also to FSUSY in refs. [20, 22]. This will allow us to compute the glueball lifetime, which together with the fragmentation function will result in a distribution of displaced vertices. The main parameter needed to compute both the fragmentation function and the decay of glueballs is their mass. This is largely determined by the infrared scale defined by the hidden glue interactions, $\Lambda_{\mathrm{IR}}$. In turn, this requires knowledge of the spectrum of states charged under the hidden glue up to a $U V$ scale. In the case of FSUSY, this means knowing the f-squark spectrum up to the scale where supersymmetry is restored, which we call $\Lambda_{\mathrm{UV}}$. This will give us the running of the hidden glue $\mathrm{SU}(3)_{B}$ coupling, $\alpha_{F}\left(q^{2}\right)$, with the $U V$ boundary condition

$$
\alpha_{F}\left(\Lambda_{\mathrm{UV}}\right)=\alpha_{s}\left(\Lambda_{\mathrm{UV}}\right),
$$

which is a $Z_{2}$-preserving UV boundary condition. In [9] the FSUSY low energy spectrum was UV-completed by a flat compact extra dimension, which was used to break supersymmetry at the compactification scale $1 / R$ by the Scherk-Schwarz boundary conditions. The f-squark soft masses then are generated by gauge loops of size $1 / R$ [35], as mentioned above. This gives a minimal way to generate the soft masses and in principle there could be additional (localized) supersymmetry breaking sources changing these soft masses. However we will assume that these would not break the relation between left- and right-handed squark masses, which is approximately given by

$$
m_{\tilde{q}_{L}}^{2} \simeq m_{\tilde{q}_{R}}^{2}+\frac{k}{4 \pi^{4}} \frac{3}{4} g^{2} \Lambda_{\mathrm{UV}}^{2}
$$

where we are using $\Lambda_{\mathrm{UV}}=1 / R$, we are neglecting terms of order $g^{\prime 2}$, and $k \simeq 2.1$ is computed in the extra dimension UV-completion [35]. Then, even if we do not make 


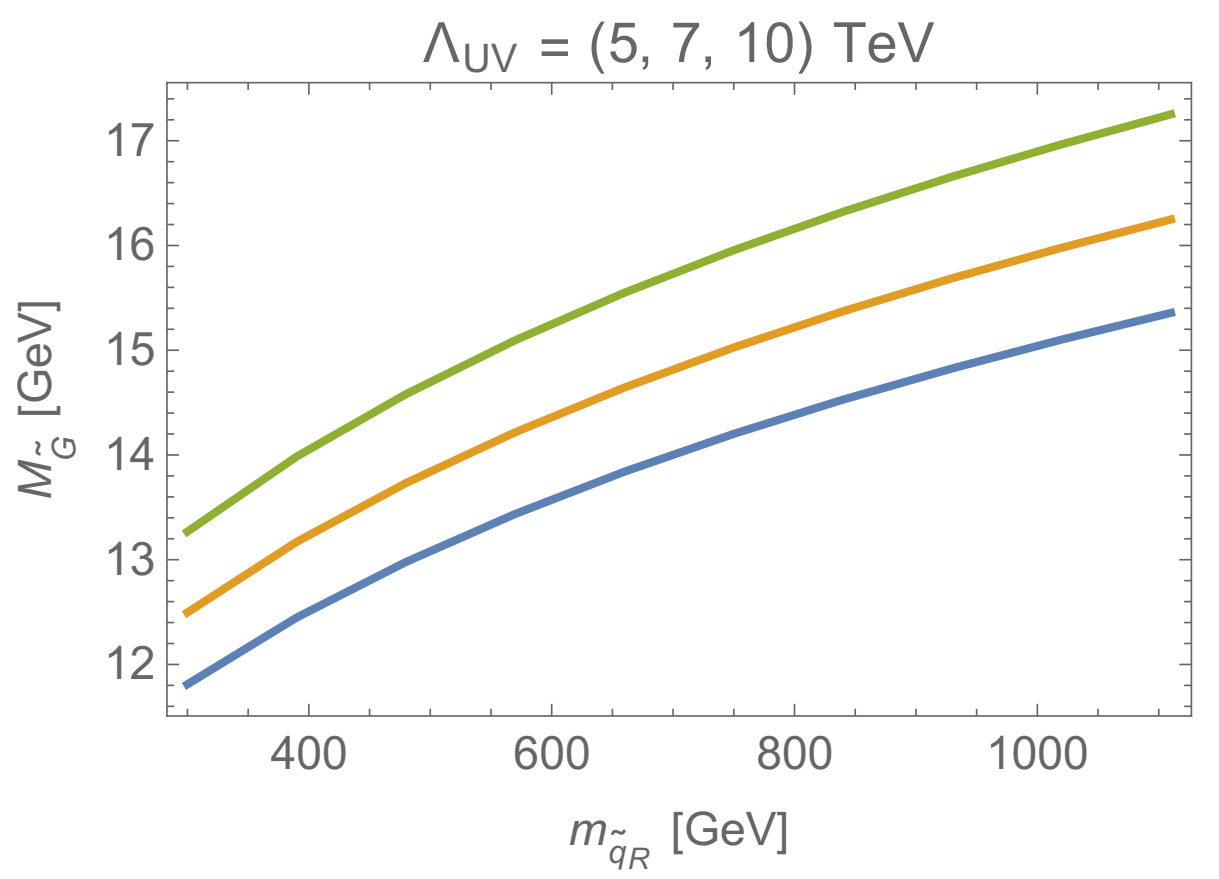

Figure 2. $0^{++}$glueball mass as a function of the right-handed f-squark mass, for (from bottom to top) $\Lambda_{\mathrm{UV}}=5,7,10 \mathrm{TeV}$.

detailed assumptions about supersymmetry breaking, we can compute $\alpha_{F}\left(q^{2}\right)$ for a given $\Lambda_{\mathrm{UV}}$ and $m_{\tilde{q}_{R}}$. We use this relation to compute the evolution of $\alpha_{F}\left(q^{2}\right)$ with the boundary condition (2.3) in order to obtain $\Lambda_{\mathrm{IR}}$. Finally, we estimate the lowest lying $0^{++}$glueball mass making use of the approximate relation

$$
M_{\tilde{G}} \simeq 7 \Lambda_{\mathrm{IR}},
$$

which has been observed to hold in QCD [12, 37-39]. The $0^{++}$state can decay back to SM states through its mixing with the Higgs boson. Heavier states, such as the $2^{++}$, the $0^{-+}$, etc., will also be produced in the folded gluon fragmentation process, but they would escape detection. We will then focus here on the fragmentation and decay properties of the $0^{++}$glueballs.

Assuming the spectrum is approximately degenerate across generations we can then compute $M_{\tilde{G}}$ as a function of $m_{\tilde{q}_{R}}$. For instance in figure 2 we show the $0^{++}$glueball mass for typical values of the UV cutoff $\Lambda_{\mathrm{UV}}$. We see that folded glueball masses are typically $(12-20) \mathrm{GeV}$ range for the interesting values of $m_{\tilde{q}_{R}}$. This gives us an estimate of the relation between $M_{\tilde{G}}$ and the typical folded gluon energy fragmenting into glueballs, which we will use in the next section to model the fragmentation function.

\section{Glueball fragmentation}

We are interested in modeling the fragmentation of energetic f-gluons (resulting from either f-squark annihilation or even from rare Higgs decays) into folded glueballs. In this section 
we lay the groundwork for this in a generic way that should mostly applied to other examples with hidden glue sectors resulting in glueballs. In general, the fragmentation function $D_{i}^{H}\left(E_{h} / E_{i}\right)$ of a given parton $i$ into a hadron $h$ obeys

$$
\frac{d \sigma(p p \rightarrow h+X)}{d E_{h}}=\sum_{i} \frac{d \sigma(p p \rightarrow i+X)}{d E_{i}} D_{i}^{h}\left(E_{h} / E_{i}\right) \frac{d E_{i}}{E_{i}},
$$

where $E_{i}$ and $E_{h}$ are the parton and hadron energies respectively, and we sum over all possible partons. Here we will consider only one parton, the folded gluon $\tilde{g}$, as well as only one hadron, the $0^{++}$glueball, or $\tilde{G}$. Fragmentation to higher mass states such as $2^{++}$ and $0^{-+}$that do not mix with the Higgs would result in missing energy since they escape detection. For our purposes, we only need to assume that the fragmentation fraction into the $0^{++}$state is constant with energy. This is a good approximation for f-gluon energies much greater than $M_{\tilde{G}}$, which is the case here. Since here we are mainly interested in the form of the distribution of displaced vertices this will not affect our results (see below), which just need only be multiplied by this constant, $r_{\tilde{G}^{0}}$. Estimates [27] using a thermal model for a pure glue theory give $r_{\tilde{G}^{0}} \simeq(0.5-0.6)$. On the other hand, the results can be affected by the presence of soft f-gluon radiation in between the two hard f-gluons. These would result in a few additional, slower moving f-glueballs. Since our calculation only considers the fragmentation from the hard f-gluons, it should be considered a lower limit to the number of displaced vertices for a given value of $r_{\tilde{G}^{0}}$.

Defining the glueball momentum fraction $x=E_{\tilde{G}} / E_{\tilde{g}}$, the availability of only one hadron for the gluon to fragment into results in the simple energy-conservation constraint

$$
\int_{x_{\min }}^{1} d x x D_{\tilde{g}}^{\tilde{G}}(x)=1
$$

where

$$
x_{\min }=\frac{M_{\tilde{G}}}{E_{\tilde{g}}} .
$$

In general, fragmentation functions are parametrized and fit to data at some energy, and then they are evolved using the DGLAP evolution equations in order to use them at different energies. This method is not available to us since we are trying to predict the glueball signal. However we can model $D_{\tilde{g}}^{\tilde{G}}(x)$ at a given input energy $s_{0}$ with a simple parametrization and impose constraints to reduce our ignorance of the fragmentation process. We start with the simple two-parameter form

$$
D_{\tilde{g}}^{\tilde{G}}(x)=N_{v}(1-x)^{\beta},
$$

where $N_{v}$ and $\beta$ are free parameters, and we should read this as $D_{\tilde{g}}^{\tilde{G}}\left(x, s_{0}\right)$. This simple expression encodes the fact that we expect the fragmentation function to fall to zero for values of $x$ not too far from 1.

However, this is not a good parametrization at low $x$. For one, the fragmentation should also fall to zero at very low $x$, where it is expected that is dominated by singularities in the gluon splitting function. The DGLAP evolution of $D_{\tilde{g}}^{\tilde{G}}(x, s)$ is given by

$$
s \frac{\partial D(x, s)}{\partial s}=\int_{x}^{1} \frac{d z}{z} \frac{\alpha_{F}(s)}{2 \pi} P_{\tilde{g} \tilde{g}}\left(z, \alpha_{F}(s)\right) D(x / z, s),
$$


where for simplicity we denoted the f-gluon to f-glueball fragmentation function as $D(x, s)$, and the evolution will only depend on the folded gluon splitting function $P_{\tilde{g} \tilde{g}}$. If we use the corresponding gluon-gluon QCD splitting function to NLO [40], the singularities in $P_{\tilde{g} \tilde{g}}$ at low $x$ in (3.5) dominate and result in [41]

$$
D(x, s) \propto \frac{1}{x} e^{-\frac{\left(\xi-\xi_{p}\right)^{2}}{2 \sigma^{2}}},
$$

where

$$
\xi \equiv \ln \frac{1}{x} \quad \xi_{p} \equiv \frac{\pi}{b \alpha_{F}(s)} \quad \sigma \equiv\left(\frac{\pi}{6 b} \sqrt{\frac{2 \pi}{C_{A} \alpha_{F}^{3}(s)}}\right)^{1 / 2},
$$

with $b$ the coefficient of the folded $\mathrm{SU}(3)_{B}$ color beta-function. The low $x$ form of the fragmentation function is valid up to about

$$
\ln \frac{1}{x} \simeq \frac{\pi}{b \alpha_{F}(s)} .
$$

Thus, we will use this form of $D(x, s)$ up to some appropriate value of $x$ and then match it to the form in (3.4) valid for larger values of $x$.

An additional constraint on the fragmentation function is that its integral results in the average glueball multiplicity $\langle n\rangle$. This is

$$
\int_{x_{\min }}^{1} d x D(x, s)=\langle n(s)\rangle .
$$

Then, a strategy to model the fragmentation of gluons into glueballs can be the following: start at an arbitrary energy scale $s_{0}$. Then choose a value of the power $\beta$ in (3.4). This choice will turn out to be equivalent to measuring the average multiplicity at $s_{0}$. Then, we match the two forms (3.4) and (3.6) at some appropriate value of $x$ and finally impose energy conservation (3.2). This procedure determines $D\left(x, s_{0}\right)$ (and also $\left\langle n\left(s_{0}\right)\right\rangle$ ). Then we can use the DGLAP evolution (3.5) to obtain $D(x, s)$ at any other desired energy scale.

It will be convenient to impose the DGLAP evolution directly on the average multiplicity. The key observation is that the average multiplicity is completely dominated by small values of $x$. As a result it is an excellent approximation to use the following form of the energy dependence for the average multiplicity [41]

$$
\langle n(s)\rangle \propto e^{\frac{4 \pi}{b} \sqrt{\frac{6 \pi}{\alpha_{F}(s)}}+\frac{1}{4} \ln \alpha_{F}(s)},
$$

where we have evaluated the exponential for zero active flavors, $n_{f}=0$. Imposing this energy dependence, and starting from a fixed value $\left\langle n\left(s_{0}\right)\right\rangle$, we fit the low and high $x$ parameterizations (3.6) and (3.4) to obtain $D(x, s)$. Imposing a value of the average multiplicity at $s_{0}$ is equivalent to choosing an initial value of $\beta$ in (3.4). The result of this process starting with $\beta=1$ at $s_{0}=200 \mathrm{GeV}$ is shown in figure 3 . We see that as the reference energy increases, the large $x$ contributions are suppressed in favor of the low $x$. In the language of the parametrization (3.4) this corresponds to increasing the power $\beta$. 


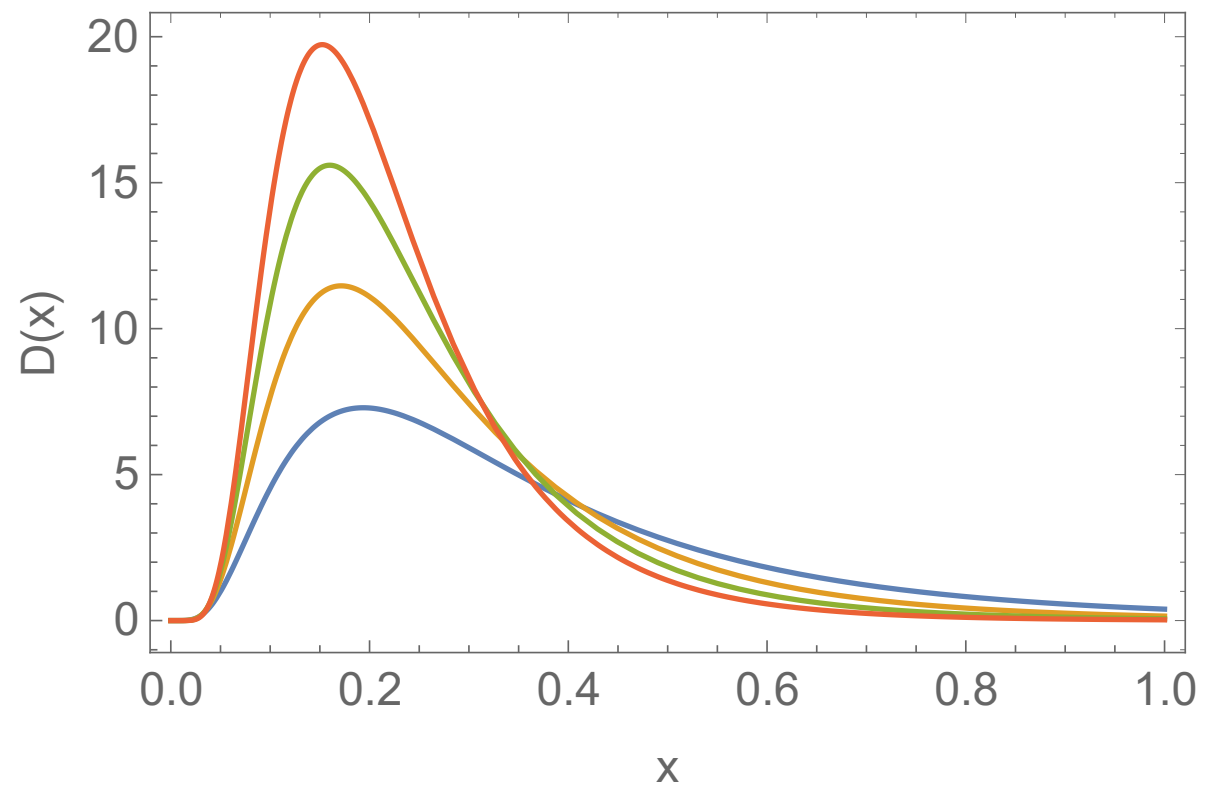

Figure 3. Fragmentation function as a function of the energy fraction $x$ for various energies. From bottom to top: $300 \mathrm{GeV}, 500 \mathrm{GeV}, 700 \mathrm{GeV}$ and $900 \mathrm{GeV}$. Here we fix the matching value as $x_{M}=0.1$, and used $M_{\tilde{G}}=15 \mathrm{GeV}$.

The corresponding average multiplicity as a function of the energy is shown in figure 4. The input value is taken to be $\left\langle n\left(s_{0}\right)\right\rangle \simeq 2.5$ for $s_{0}=200 \mathrm{GeV}$, which corresponds to choosing $\beta=1$ for large $x$ at that energy. We choose $s_{0}$ to have a value safely higher than any possible value for $M_{\tilde{G}}$. Although this value for the parameter $\beta$ at $s_{0}$, and therefore the normalization of the average multiplicity is a priori arbitrary, it reflects the simplest parametrization of the large momentum fraction region, i.e. a linearly falling fragmentation.

The choice of $\beta \simeq 1$ for $s_{0}$ is also suggested by the similarity observed between the glueball wave-function and that of the $\pi^{0}$, as obtained in QCD sum rules [42, 43]. This implies that the fragmentation function of a gluon into a glueball is similar to the one of valence quarks into $\pi^{0}$, as it is pointed out in [44]. The relevant fits to the parametrization (3.4) valid at large $x$ can be found, for instance, in [45], where we see that $\beta \simeq 1$ correctly reproduces the average multiplicity. The more sophisticated parameterizations [46] currently used, for instance, for the pion fragmentation function are next-to-leading order and are designed to be valid for both small and high momentum fractions. Ultimately, this input parameter can be fixed by using a Monte Carlo simulation of the parton shower in a quarkless model of QCD, i.e. a simulation of the F-SUSY low energy theory. This would greatly reduce the uncertainty in the model for $D(x)$. In the meantime, having a model for the fragmentation allows us to make estimates of the qualitative behavior of the signal in detectors.

One remaining arbitrary parameter of the model is the value of the momentum fraction $x_{M}$ used to match the low and high $x$ behaviors of the fragmentation function. In figure 5 


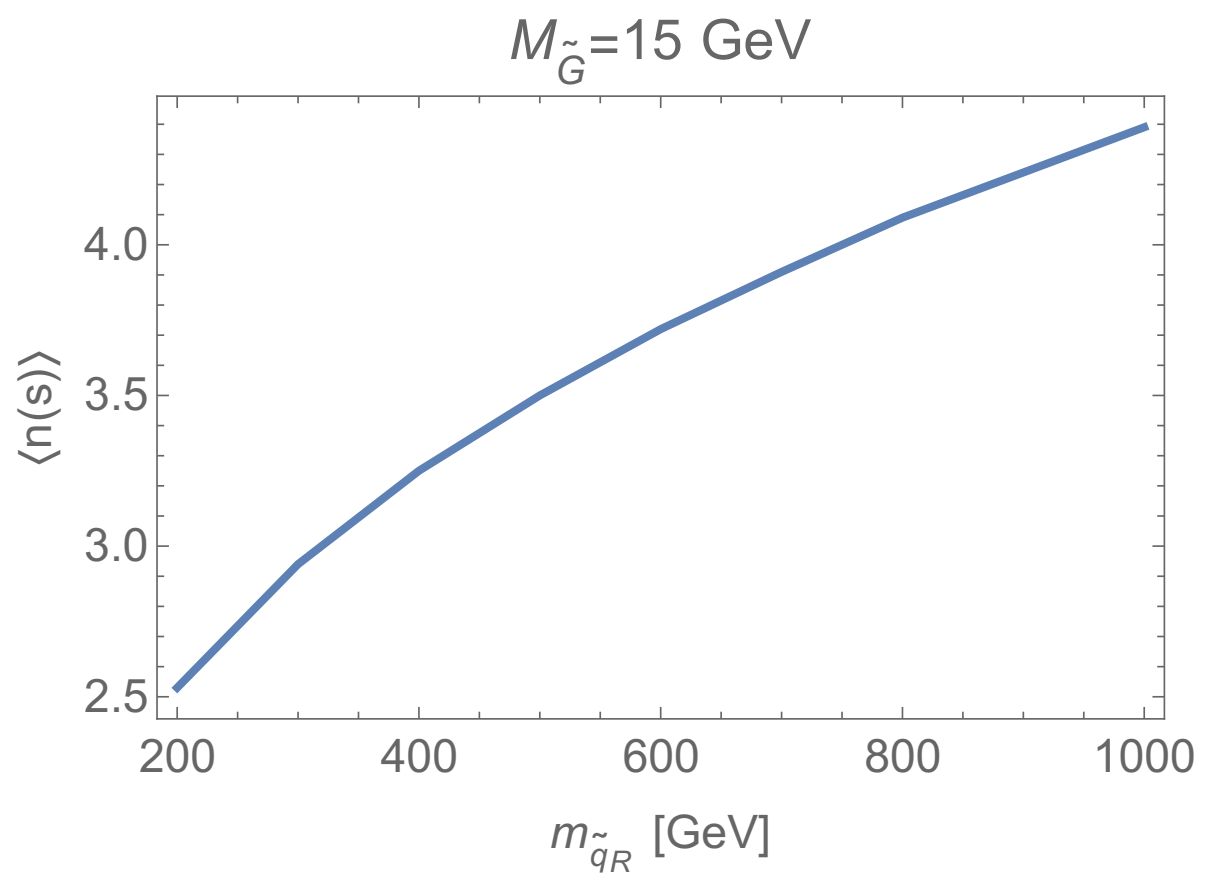

Figure 4. Average multiplicity of glueballs as a function of energy. Here we used $x_{M}=0.1$ and $M_{\tilde{G}}=15 \mathrm{GeV}$.

we vary its value in order to study the sensitivity of the result to this choice. We fix the gluon energy at $500 \mathrm{GeV}$ and use for the matching momentum fraction three values $x_{M}=0.07,0.10,0.13$. From the figure we see that the systematic uncertainty associated with this parameter is not too large, especially if we consider this as a useful model of the fragmentation function that will allow us to simulate the distribution of the displaced vertices in a detector. To quantify the uncertainty we look at the average multiplicity resulting from these three choices: $\langle n(500 \mathrm{GeV})\rangle=3.60,3.50,3.36$, and see that it is not such a large variation. As we will see in the next section, the largest uncertainty in the prediction for the distribution of displaced vertices from glueball production and decays will be the glueball mass.

\section{Displaced vertices from glueballs}

Armed with the gluon-to-glueball fragmentation function $D(x, s)$ we are now in the position to predict the distribution of displaced vertices generated once they decay back to SM states. Assuming that the gluon fragments into a glueball promptly, the number of displaced vertices as a function of the decay length $L$ is given by

$$
N_{\mathrm{dv}}(L)=\int_{x_{\min }}^{1} d x D(x, s)\left(1-e^{L / \bar{L}_{\tilde{G}}(x)}\right),
$$

where we have defined

$$
\bar{L}_{\tilde{G}}(x) \equiv c \tau_{\tilde{G}} \frac{x}{x_{\min }},
$$




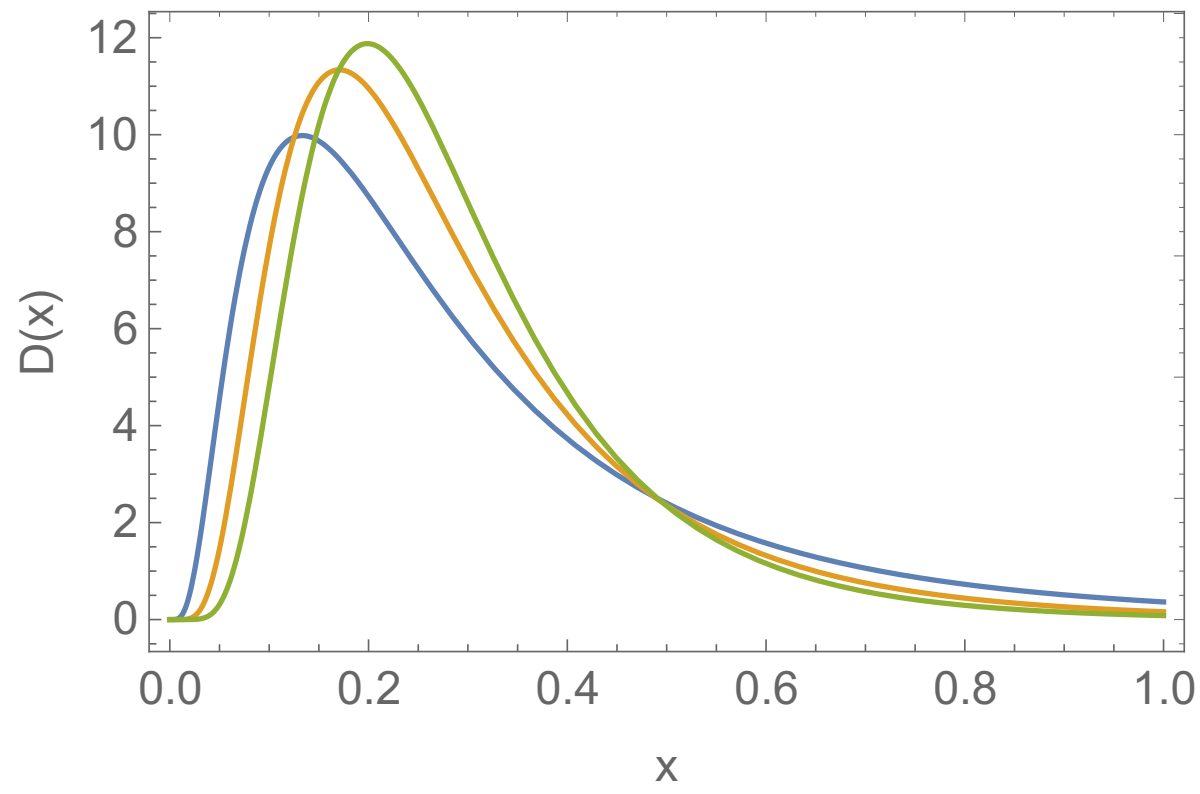

Figure 5. Fragmentation function as a function of the energy fraction $x$ for $500 \mathrm{GeV}$, for various choices of matching value $x_{M}$. From bottom to top: $x_{M}=0.07,0.10$ and 0.13 .

which is the decay length determined by the glueball lifetime $\tau_{\tilde{G}}$. As seen in (4.2), it depends on the energy fraction of the glueball, $x / x_{\min }$ which is simply the $\gamma$ factor. In order to compute the distribution in (4.1) we need to know the glueball lifetime, which depends crucially on details of the model since $\tau_{\tilde{G}}$ is typically determined by a higher dimensional operator mixing the $0^{++}$glueball with the Higgs boson. This is given by $[12,26]$

$$
\mathcal{O} \sim \frac{\alpha_{F}}{4 \pi}|H|^{2} \operatorname{Tr}\left[\tilde{G}_{\mu \nu} \tilde{G}^{\mu \nu}\right],
$$

with $\tilde{G}_{\mu \nu}$ the hidden gluon field strength. For the case of FSUSY the coefficient multiplying (4.3) is given by [20]

$$
\frac{1}{48 \pi} \frac{y_{t}^{2}}{m_{\tilde{t}}^{2}}
$$

where $y_{t}$ is the top Yukawa coupling and it is assumed that $m_{\tilde{t}_{1}}=m_{\tilde{t}_{2}}=m_{\tilde{t}}$. Then, the resulting glueball decay width to a given SM final state is

$$
\Gamma(\tilde{G} \rightarrow \mathrm{SM})=\left(\frac{\alpha_{F}}{48 \pi} \frac{y_{t}^{2}}{m_{\tilde{t}}^{2}} \frac{v}{m_{h}^{2}-M_{\tilde{G}}^{2}}\right)^{2} f_{\tilde{G}}^{2} \Gamma(h \rightarrow \mathrm{SM}) .
$$

Here, the Higgs decay width to the SM final state corresponds to that of an off-shell Higgs, and it must be evaluated with the replacement $m_{h} \rightarrow M_{\tilde{G}}$. Also in (4.5) we defined the hadronic matrix element creating the glueball from the gluon operator defined by

$$
\left\langle 0\left|\operatorname{Tr}\left[\tilde{G}_{\mu \nu} \tilde{G}^{\mu \nu}\right]\right| \tilde{G}\right\rangle \equiv f_{\tilde{G}},
$$

with $f_{\tilde{G}}$ the glueball decay constant. Lattice studies result in [47]

$$
4 \pi \alpha_{F} f_{\tilde{G}} \simeq 2.3 M_{\tilde{G}}^{3}
$$


Since the Higgs decay width back to the SM goes like $\sim M_{\tilde{G}}$ we see that the lifetime scales as

$$
\tau_{\tilde{G}} \sim \frac{m_{\tilde{t}}^{4}}{M_{\tilde{G}}^{7}}
$$

Thus, the glueball lifetime is extremely sensitive to the details resulting in the glueball mass. To illustrate this point we show the distribution of displaced vertices as a function of the distance to the glueball production point for three values of the glueball mass: $M_{\tilde{G}}=15,20$ and $30 \mathrm{GeV}$ in figures 6 and 7 . In figure 6 we plot the number of displaced vertices as a function of the distance from the production point for $M_{\tilde{G}}=15 \mathrm{GeV}$, for three values of the masses of the squarks whose annihilation gives rise to the hard folded gluons hadronizing into glueballs. The vertical lines correspond to the approximate positions of the different sections of an idealized version of the ATLAS detector ${ }^{1}$ starting from the trackers, then the electromagnetic and hadronic calorimeters and all the way to the muon systems going out to about 10 meters. The horizontal lines are the average multiplicities for each value of the squark mass. For instance, we see that even for relatively small masses such as $m_{\tilde{q}_{R}}=300 \mathrm{GeV}$ an important fraction of the displaced vertices occur in or beyond the muon system (about $1 / 3$ ), with about half of the events in the calorimeters. For the larger squark masses, most of the events within the detector will be in the muon system, although the majority of the displaced vertices will be beyond it.

The situation changes drastically as the glueball mass is increased. In figure 7 we show the distribution of displaced vertices for $M_{\tilde{G}}=20 \mathrm{GeV}$ (left) and $M_{\tilde{G}}=30 \mathrm{GeV}$ (right). For instance, for $M_{\tilde{G}}=20 \mathrm{GeV}$ we see that for $m_{\tilde{q}_{R}}=300 \mathrm{GeV}$ almost all displaced vertices would be in the electromagnetic calorimeter. For the most massive case, $m_{\tilde{q}_{R}}=700 \mathrm{GeV}$, almost $2 / 3$ of the events are inside the detector, with roughly half of them in the calorimeters and the other half in the muon system. For $M_{\tilde{G}}=30 \mathrm{GeV}$, we see that all of the events are inside the detector even for the larger squark masses considered, with a large fraction in the electromagnetic calorimeter. Thus, even relatively small changes in the glueball mass would lead to a very different distribution of displaced vertices. This calls for developing strategies for searching for displaced vertices in all segments of the LHC detectors. In particular, since folded glueballs in FSUSY decay preferentially to a pair of b quarks, detections of these should be made possible in all detector components, not just the hadronic calorimeter.

There can be other theories with glueballs or other low lying states with long lifetimes induced, for instance, by higher dimensional operators such as in FSUSY. Although some of the details may be different, the procedure to obtain the fragmentation function and then the distribution of displaced vertices is analogous to what was done above.

Depending on the folded gluon energy resulting from the f-squark annihilation, as well as on the glueball mass, the number of displaced vertices inside the detector on each side of the event can be larger than one. This presents a challenge for the searches for these events at ATLAS and CMS. The modeling of the glueball fragmentation function allows us

\footnotetext{
${ }^{1}$ This is just chosen for illustration. We do not attempt here a realistic detector simulation. An analogous study can be done for an idealized version of CMS.
} 


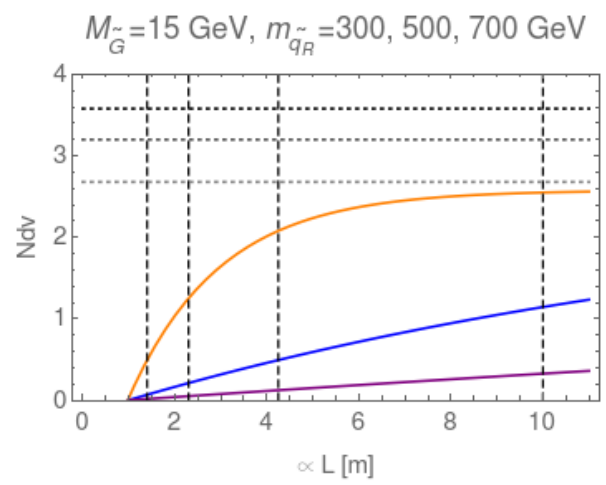

Figure 6. Distribution of displaced vertices for $m_{\tilde{q}}=300,500$ and $700 \mathrm{GeV}$, and $M_{\tilde{G}}=15 \mathrm{GeV}$. The vertical axis, $\mathrm{Ndv}$ is the number of displaced vertices as a function of distance $\mathrm{L}$ from the primary, normalized by $r_{\tilde{G}^{0}}$, the f-gluon fragmentation fraction into the $0^{++}$glueball. The vertical lines indicate approximate boundaries of the inner tracker, electromagnetic calorimeter, hadronic calorimeter and muon system of the ATLAS detector. The horizontal dotted lines correspond to the average multiplicity for each of the three cases for the squark masses, from bottom to top: $300 \mathrm{GeV}, 500 \mathrm{GeV}$ and $700 \mathrm{GeV}$.
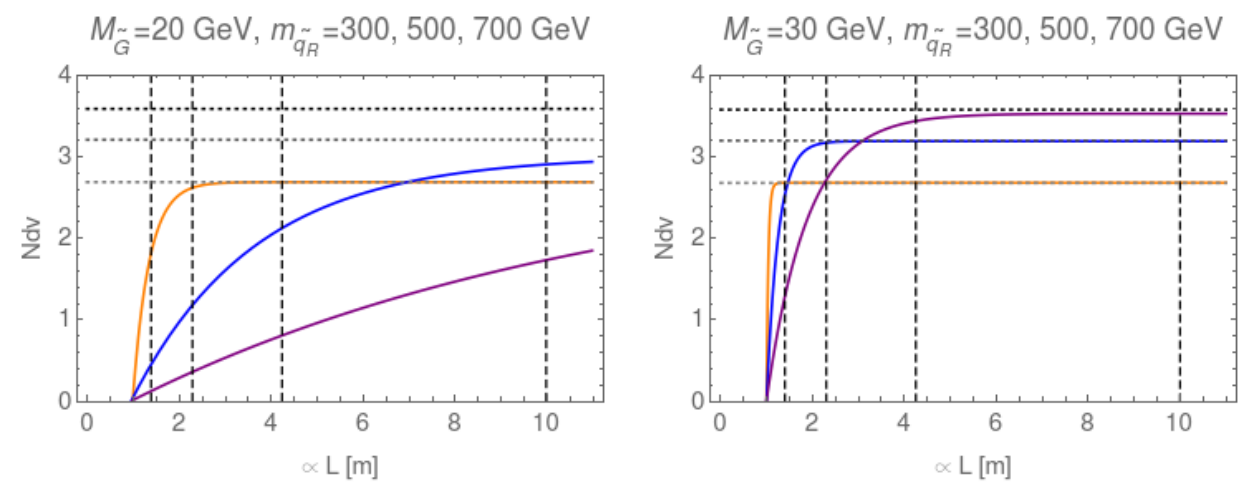

Figure 7. Same as figure 6, but for $M_{\tilde{G}}=20 \mathrm{GeV}$ (left) and $M_{\tilde{G}}=30 \mathrm{GeV}$ (right).

to have a more detailed knowledge of the multiplicity and of the location of the displaced vertices as a function of the parameters of the models.

Searches for long-lived particles need to include the possibility of having the kind of secondary vertex topology that appears in these models. For instance in [48] the ATLAS collaboration considers three kinds of models: a singlet scalar decaying to two long-lived scalars each decaying to two jets, stealth SUSY and a hidden-valley model where a $Z^{\prime}$ decays to several long-lived scalar. In the first two cases the signal contains two displaced vertices with two-jet pairs which are somewhat back to back. This search requires two displaced vertices (either in the inner detector or in the muon system or one in each). This situation is reproduced in the folded gluon fragmentation into glueballs for rather light f-squarks and small glueball masses (e.g. top curve in figure 6, for $m_{\tilde{q}_{R}}=300 \mathrm{GeV}$ ), although the decays tend to happen in the calorimeters instead. On the other hand, for larger values of $m_{\tilde{q}_{R}}$ (bottom two curves of figure 6), there maybe even less than two 
displaced vertices inside the detector. In these cases it is important to look for large values of missing transverse energy.

When there are more than two displaced vertices in the event such as in the second half of the top curve of figure 6 or in figure 7 , the high multiplicity may result in failing isolation cuts. Although in these cases it is possible that some of the decays are outside the detector and give large missing transverse energy, it may be also possible to make use of the topology of the event which is still dominated by two well separated clusters almost back to back resulting from the very energetic folded gluons. A combination of the reconstruction of some of the displaced vertices, the topology of the multi-jet event and some missing $E_{T}$ may be needed to isolate these events from background. In any case, an increased efficiency for the reconstruction of displaced vertices in all detector segments might be necessary, particularly in the muon system.

On the other hand, the CMS collaboration [49] has done a study using a benchmark model with the pair production of two long-lived scalars decaying to jets similar to the first model considered by ATLAS in [48]. However, in this analysis displaced vertices are not fully reconstructed but identified by "displaced jets" variables. These are used as selection criteria for jets, and then each event selected will contain a number of jets tagged as displaced. This study is, in principle, not limited by the number of tagged jets to a fix number of displaced vertices and could be an adequate framework for the case with many folded glueballs. More detailed studies following the experimental strategies and making use of the information from fragmentation are left for future work.

\section{Conclusions}

Theories with hidden strong sectors may result in low lying states without interactions with SM matter, but which can decay back to the SM via higher dimensional operators. In this paper we studied the case of FSUSY glueballs produced through the annihilation of electro-weakly produced folded squark pairs. The f-squarks annihilate into hard f-gluons which then hadronize into f-glueballs. Although in general f-gluons would hadronize into several f-glueball states we have only considered the lowest lying one, $0^{++}$, since it can be detected via its mixing with the Higgs. The fragmentation fraction into $0^{++}, r_{\tilde{G}^{0}}$, is expected to be a constant with energy for the case we study here. Our results for the number of displaced vertices should then be multiplied by it, which can be estimated using a thermal model to be $r_{\tilde{G}^{0}} \simeq(0.5-0.6)$.

We have modeled the glueball fragmentation process obtaining a fragmentation function. This was achieved by appropriately parameterizing the low and high momentum fraction regions of the fragmentation function. At low momentum fractions the parametrization is chosen to be compatible with the known behavior of the average multiplicity in (3.10). A simple parametrization for the region of high momentum fraction, that of (3.4), is used as initial input. Imposing energy conservation through (3.2) and the average multiplicity (3.9) we fix all parameters at a given input energy $s_{0}$ except one. We take this to be the value of $\beta$ in (3.4) at the input energy $s_{0}$, which determines $\left\langle n\left(s_{0}\right)\right\rangle$ by fixing the overall energy-independent constant in (3.10). Once this is done, the energy dependence of the 
fragmentation function $D(x, s)$ is fixed by DGLAP evolution. This is taken into account by imposing (3.10) on the integral of the fragmentation function $D(x, s)$ resulting in the average multiplicity, equation (3.9). This translates into a DGLAP evolution of the parameters for $D(x, s)$ in both the small as well as the large momentum fraction regimes. The scale where these two parameterizations are matched, $x_{M}$ is also free parameter but is approximately given by (3.8), which determines the validity of the low momentum-fraction region. As seen in figure 5, variation of $x_{M}$ about a reasonable range compatible with (3.8) does not result in dramatic changes in the fragmentation function. Thus, the main parametric freedom can be traced back to the overall normalization of (3.10), or the reference value of the parameter $\beta$ in (3.4) for a given energy $s_{0}$. We have used $\beta=1$ for $s_{0}=200 \mathrm{GeV}$, which results in reasonable values of the average multiplicity. This linear parametrization for the reference value is inspired by a similar one successfully used for the neutral pion. Ultimately, this arbitrariness can be removed by using a parton shower simulation in FSUSY to compute the average multiplicity at the reference energy $s_{0}$. This would result in a much less uncertain glueball fragmentation function, which can then be used with more confidence to extract model information from events with displaced vertices.

In section 4 we have made use of these folded glueball fragmentation functions to obtain the distribution of displaced vertices at a typical LHC experiment resulting from the production and subsequent annihilation of the lightest matter states in FSUSY, a pair of right-handed f-squarks. The annihilation into a pair of hard f-gluons and their fragmentation into glueballs would provide the first signal of FSUSY that is associated with the existence of a hidden glue sector, a critical component of the theory. Our results show that it is likely that there will be more than one displaced vertex per side of the event. Also, depending on the details of the theory and most dramatically on the glueball mass, the distribution of these vertices can fall almost anywhere in a typical LHC detector. In figures 6 and 7 we exemplified this point by choosing three values of the glueball mass, and various values for the right-handed squark masses that define the hard f-gluon energy. We see that for the lighter glueball mass preferred by our estimates, i.e. $M_{\tilde{G}}=15 \mathrm{GeV}$, most of the displaced vertices occur in the outer elements of the detector, such as the muon systems or even beyond, as seen in figure 6. On the other hand, since the computation of the glueball mass is rather uncertain, if we allow for somewhat larger values of $M_{\tilde{G}}$, the situation changes dramatically and most of the events are contained in the inner segments of the detector. It will be important then to develop searches capable of observing multivertex events in virtually any part of the detector, and possibly in more than one element at the same time.

Since the cross sections producing these events are below a $1 \mathrm{fb}$ (see figure 1) large luminosities will be necessary to see this physics above background. This study is a step in the direction of modeling the ensuing multi-displaced vertex signals. Next, it will be necessary to fix further the parameters of the glueball fragmentation function by "measuring" the average multiplicity at one value of the energy in a Monte Carlo shower simulation. This would put our results for $D(x, s)$ on a better footing. Finally, a better modeling of the glueballs resulting from soft f-gluon radiation in the event after f-squark annihilation, would be helpful before a detailed simulation for high luminosities is undertaken. 


\section{Acknowledgments}

The authors thank Z. Chacko, D. Curtin and R. Sundrum for helpful discussions. They acknowledge the support of FAPESP, CNPq and CAPES.

Open Access. This article is distributed under the terms of the Creative Commons Attribution License (CC-BY 4.0), which permits any use, distribution and reproduction in any medium, provided the original author(s) and source are credited.

\section{References}

[1] ATLAS collaboration, Observation of a new particle in the search for the Standard Model Higgs boson with the ATLAS detector at the LHC, Phys. Lett. B 716 (2012) 1 [arXiv: 1207.7214] [INSPIRE].

[2] CMS collaboration, Observation of a new boson at a mass of $125 \mathrm{GeV}$ with the CMS experiment at the LHC, Phys. Lett. B 716 (2012) 30 [arXiv:1207.7235] [INSPIRE].

[3] S.P. Martin, A supersymmetry primer, Adv. Ser. Direct. High Energy Phys. 18 (1998) 1 [hep-ph/9709356] [INSPIRE].

[4] I.J.R. Aitchison, Supersymmetry and the MSSM: An elementary introduction, hep-ph/0505105 [INSPIRE].

[5] B. Bellazzini, C. Csáki and J. Serra, Composite Higgses, Eur. Phys. J. C 74 (2014) 2766 [arXiv: 1401.2457] [INSPIRE].

[6] G. Panico and A. Wulzer, The Composite Nambu-Goldstone Higgs, Lect. Notes Phys. 913 (2016) 1 [arXiv: 1506.01961] [INSPIRE].

[7] Z. Chacko, H.-S. Goh and R. Harnik, The Twin Higgs: Natural electroweak breaking from mirror symmetry, Phys. Rev. Lett. 96 (2006) 231802 [hep-ph/0506256] [INSPIRE].

[8] H. Cai, H.-C. Cheng and J. Terning, A Quirky Little Higgs Model, JHEP 05 (2009) 045 [arXiv: 0812.0843] [INSPIRE].

[9] G. Burdman, Z. Chacko, H.-S. Goh and R. Harnik, Folded supersymmetry and the LEP paradox, JHEP 02 (2007) 009 [hep-ph/0609152] [INSPIRE].

[10] N. Craig, S. Knapen and P. Longhi, Neutral Naturalness from Orbifold Higgs Models, Phys. Rev. Lett. 114 (2015) 061803 [arXiv:1410.6808] [INSPIRE].

[11] N. Craig, S. Knapen and P. Longhi, The Orbifold Higgs, JHEP 03 (2015) 106 [arXiv: 1411.7393] [INSPIRE].

[12] N. Craig, A. Katz, M. Strassler and R. Sundrum, Naturalness in the Dark at the LHC, JHEP 07 (2015) 105 [arXiv: 1501.05310] [INSPIRE].

[13] R. Barbieri, D. Greco, R. Rattazzi and A. Wulzer, The Composite Twin Higgs scenario, JHEP 08 (2015) 161 [arXiv: 1501.07803] [INSPIRE].

[14] M. Low, A. Tesi and L.-T. Wang, Twin Higgs mechanism and a composite Higgs boson, Phys. Rev. D 91 (2015) 095012 [arXiv:1501.07890] [INSPIRE].

[15] T. Gherghetta, M. Nguyen and Z. Thomas, Neutral Naturalness with Bifundamental Gluinos, Phys. Rev. D 94 (2016) 115008 [arXiv:1610.00342] [INSPIRE]. 
[16] N. Craig, S. Knapen, P. Longhi and M. Strassler, The Vector-like Twin Higgs, JHEP 07 (2016) 002 [arXiv: 1601.07181] [InSPIRE].

[17] J. Serra and R. Torre, Neutral naturalness from the brother-Higgs model, Phys. Rev. D 97 (2018) 035017 [arXiv: 1709.05399] [INSPIRE].

[18] T. Cohen, N. Craig, G.F. Giudice and M. Mccullough, The Hyperbolic Higgs, JHEP 05 (2018) 091 [arXiv: 1803.03647] [INSPIRE].

[19] H.-C. Cheng, L. Li, E. Salvioni and C.B. Verhaaren, Singlet Scalar Top Partners from Accidental Supersymmetry, JHEP 05 (2018) 057 [arXiv: 1803.03651] [INSPIRE].

[20] D. Curtin and C.B. Verhaaren, Discovering Uncolored Naturalness in Exotic Higgs Decays, JHEP 12 (2015) 072 [arXiv: 1506.06141] [INSPIRE].

[21] G. Burdman, Z. Chacko, H.-S. Goh, R. Harnik and C.A. Krenke, The Quirky Collider Signals of Folded Supersymmetry, Phys. Rev. D 78 (2008) 075028 [arXiv:0805.4667] [INSPIRE].

[22] Z. Chacko, D. Curtin and C.B. Verhaaren, A Quirky Probe of Neutral Naturalness, Phys. Rev. D 94 (2016) 011504 [arXiv: 1512.05782] [InSPIRE].

[23] M.J. Strassler and K.M. Zurek, Echoes of a hidden valley at hadron colliders, Phys. Lett. B 651 (2007) 374 [hep-ph/0604261] [INSPIRE].

[24] M.J. Strassler and K.M. Zurek, Discovering the Higgs through highly-displaced vertices, Phys. Lett. B 661 (2008) 263 [hep-ph/0605193] [INSPIRE].

[25] T. Han, Z. Si, K.M. Zurek and M.J. Strassler, Phenomenology of hidden valleys at hadron colliders, JHEP 07 (2008) 008 [arXiv: 0712.2041] [INSPIRE].

[26] J.E. Juknevich, D. Melnikov and M.J. Strassler, A Pure-Glue Hidden Valley I. States and Decays, JHEP 07 (2009) 055 [arXiv:0903.0883] [INSPIRE].

[27] J.E. Juknevich, Phenomenology of pure-gauge hidden valleys at Hadron colliders, Ph.D. Thesis, Rutgers University, Piscataway, U.S.A. (2010).

[28] D. Curtin et al., Long-Lived Particles at the Energy Frontier: The MATHUSLA Physics Case, arXiv: 1806.07396 [INSPIRE].

[29] A. Sopczak, SUSY (ATLAS), in 6th International Conference on New Frontiers in Physics (ICNFP 2017) Kolymbari, Crete, Greece, August 17-26, $201 \%$.

[30] CMS collaboration, F. Lacroixon, SUSY searches with the CMS detector, Nuovo Cim. C 40 (2017) 189 [INSPIRE].

[31] J. Kang and M.A. Luty, Macroscopic Strings and 'Quirks' at Colliders, JHEP 11 (2009) 065 [arXiv: 0805.4642] [INSPIRE].

[32] R. Harnik, G.D. Kribs and A. Martin, Quirks at the Tevatron and Beyond, Phys. Rev. D 84 (2011) 035029 [arXiv: 1106.2569] [INSPIRE].

[33] R. Fok and G.D. Kribs, Chiral Quirkonium Decays, Phys. Rev. D 84 (2011) 035001 [arXiv:1106.3101] [INSPIRE].

[34] G. Burdman, Z. Chacko, R. Harnik, L. de Lima and C.B. Verhaaren, Colorless Top Partners, a $125 \mathrm{GeV}$ Higgs and the Limits on Naturalness, Phys. Rev. D 91 (2015) 055007 [arXiv: 1411.3310] [INSPIRE].

[35] A. Delgado, A. Pomarol and M. Quirós, Supersymmetry and electroweak breaking from extra dimensions at the TeV scale, Phys. Rev. D 60 (1999) 095008 [hep-ph/9812489] [InSPIRE]. 
[36] ATLAS collaboration, Search for new resonances in $W \gamma$ and $Z \gamma$ final states in pp collisions at $\sqrt{s}=8 \mathrm{TeV}$ with the ATLAS detector, Phys. Lett. B 738 (2014) 428 [arXiv:1407.8150] [INSPIRE].

[37] C.J. Morningstar and M.J. Peardon, The glueball spectrum from an anisotropic lattice study, Phys. Rev. D 60 (1999) 034509 [hep-lat/9901004] [InSPIRE].

[38] Y. Chen et al., Glueball spectrum and matrix elements on anisotropic lattices, Phys. Rev. D 73 (2006) 014516 [hep-lat/0510074] [INSPIRE].

[39] M. Gockeler et al., A determination of the Lambda parameter from full lattice QCD, Phys. Rev. D 73 (2006) 014513 [hep-ph/0502212] [INSPIRE].

[40] W. Furmanski and R. Petronzio, Singlet Parton Densities Beyond Leading Order, Phys. Lett. B 97 (1980) 437 [INSPIRE].

[41] R. Keith Ellis, W. James Stirling and B.R. Webber, QCD and collider physics, Camb. Monogr. Part. Phys. Nucl. Phys. Cosmol. 8 (1996) 1.

[42] A.B. Wakely and C.E. Carlson, Pseudoscalar glueball wave functions from QCD sum rules, Phys. Rev. D 45 (1992) 338 [INSPIRE].

[43] A.B. Wakely and C.E. Carlson, Two photon production of pseudoscalar glueballs, Phys. Rev. D 45 (1992) 1796 [INSPIRE].

[44] P. Roy and K. Sridhar, A new proposal for glueball exploration in hard gluon fragmentation, JHEP 07 (1999) 013 [hep-ph/9902400] [INSPIRE].

[45] P. Chiappetta, M. Greco, J.P. Guillet, S. Rolli and M. Werlen, Next-to-leading order determination of pion fragmentation functions, Nucl. Phys. B 412 (1994) 3 [hep-ph/9301254] [INSPIRE].

[46] Particle Data Group collaboration, C. Patrignani et al., Review of Particle Physics, Chin. Phys. C 40 (2016) 100001 [inSPIRE].

[47] H.B. Meyer, Glueball matrix elements: a lattice calculation and applications, JHEP 01 (2009) 071 [arXiv:0808.3151] [INSPIRE].

[48] ATLAS collaboration, Search for long-lived, weakly interacting particles that decay to displaced hadronic jets in proton-proton collisions at $\sqrt{s}=8 \mathrm{TeV}$ with the ATLAS detector, Phys. Rev. D 92 (2015) 012010 [arXiv:1504.03634] [INSPIRE].

[49] CMS collaboration, Search for new long-lived particles at $\sqrt{s}=13 \mathrm{TeV}$, Phys. Lett. B 780 (2018) 432 [arXiv: 1711.09120] [INSPIRE]. 